\section{AN UNEXPECTED CAUSE OF PREMATURE MENOPAUSE}

Helen Keeman $^{1,2}$, Maryam Nejat ${ }^{1}$, Kym Drake $^{2}$, Kylie Drake $^{2}$, Richard King $^{2}$, Rachael van der Griend

${ }^{1}$ Anatomical Pathology Department, Canterbury Health Laboratories, Christchurch Public Hospital, New Zealand; and

${ }^{2}$ Genetics Department, Canterbury Health Laboratories, Christchurch Public Hospital, New Zealand

Presenting complaint: A 42-year-old female presented with 6 weeks of bloody vaginal discharge, as well a history of abrupt early menopause 2 years prior, with no post-menopausal symptoms. Her last known pregnancy was 14 years ago.

Investigations and results: The patient had a serum bHCG level of 59,000. An ultrasound scan revealed a $42 \mathrm{~mm}$ intrauterine mass with a complex cystic structure, and she underwent a total abdominal hysterectomy for suspected gestational trophoblastic neoplasia. Histology showed a $90 \mathrm{~mm}$ mixed choriocarcinoma and placental site trophoblastic tumour (PSTT) arising in the uterus and extending into the proximal cervix. Immunohistochemical stains supported this diagnosis. The tumour underwent quantitative fluorescent PCR analysis (QF-PCR), and was found to be gestational in origin.

Case discussion: We will review the clinical, histological and immunohistochemical features of choriocarcinoma and placental site trophoblastic tumour. We will demonstrate how QF-PCR analysis may be used to determine whether a tumour is gestational, ${ }^{1}$ and discuss the clinical significance of this finding for this patient.

\section{Reference}

1. O'Neill C, Houghton F, Clarke J, et al. Uterine gestational choriocarcinoma developing after a long latent period in a postmenopausal woman: the value of DNA polymorphism studies. Int J Surg Pathol 2008; 16 : $226-9$.

\section{A STUDY OF GATA-3 IN MALIGNANT PLEURAL MESOTHELIOMA}

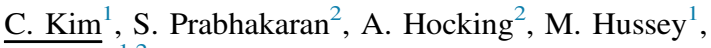
S. Klebe ${ }^{1,2}$

${ }^{1}$ Department of Surgical Pathology, SA Pathology at Flinders Medical Centre, Adelaide, SA, Australia; and ${ }^{2}$ Department of Anatomical Pathology, College of Medicine and Public health, Flinders University, Adelaide, SA, Australia

Background: Malignant pleural mesothelioma is an asbestosassociated tumour with high incidence in Australia. ${ }^{1}$ Diagnosis and prognostication remain difficult. ${ }^{2} \mathrm{We}$ investigated the usefulness of GATA-3 and BAP-1 immunohistochemistry for diagnosis and prognostication in pleural mesothelioma.

Methods: We scored immunohistochemical labelling for GATA-3 and BAP-1 in 116 malignant pleural mesotheliomas and correlated labelling with clinical parameters, histological subtypes and survival. SPSS version 23 statistical software was used for analyses.

Results: Median age at diagnosis was 74.5 years (93 males:23 females). GATA-3 was positive in $56 / 116(48 \%)$ cases $(23 / 62$ (37\%) epithelioid, 22/32 (69\%) sarcomatoid and 11/22 (50\%) biphasic). $73 \%$ of the biphasic subtype showed either positivity or negativity on both components. There was a trend to better survival in GATA-3 positive cases with epithelioid histology $(p=0.081)$ and among women $(p=0.001)$. GATA-3 was not an independent prognostic marker on multivariate analysis. BAP-1 loss was seen in 44/106 (42\%) and was associated with the epithelioid subtype $(p<0.001)$ on Chi square analysis but not independently with survival.

Conclusion: Almost half our cohort of malignant pleural mesotheliomas labelled for GATA-3 with highest incidences among sarcomatoid mesotheliomas. Discordance in GATA-3 labelling among the components of the biphasic subtype was unexpected. Neither GATA-3 nor BAP-1 showed clear association with survival.

References

1. Australian Institute of Health and Welfare (AIHW). Mesothelioma in Australia 2018. Canberra: AIHW, 2019.

2. Cornelissen R, Aerts J. Biomarkers in malignant mesothelioma-an unfulfilled need or a waste of resources? J Thorac Dis 2018; 10 (Suppl 9): S1084-7.

\section{SIMPLE YET RARE: SIMPLE PANCREATIC CYST IN AN ADULT - A CASE REPORT}

Se Mi Kim ${ }^{1}$, Kai Y. Chau ${ }^{1}$, Ole Steen Bjerring ${ }^{2}$, Paul Restall ${ }^{1}$

${ }^{1}$ Anatomical Pathology, LabPlus, Auckland City Hospital, Auckland, New Zealand; and ${ }^{2}$ Hepatopancreaticobiliary Surgery, Auckland City Hospital, Auckland, New Zealand

Pancreatic cysts (PC) are very common, and with the increased use of cross-sectional imaging the incidence of PC seems to increase too. Simple pancreatic cysts are very rare and most are found in children in the context of other congenital anomalies, such as von Hippel-Lindau disease and polycystic kidney disease. Here, we report a case of a simple pancreatic cyst in a 69-year-old female. A $6 \mathrm{~cm}$ cyst in the tail of the pancreas was incidentally discovered on a computerised tomography colonography. Due to the size and morphological pattern on the imaging mimicking a mucinous cystic neoplasm, laparoscopic spleen-preserving distal pancreatectomy was performed. Macroscopically the pancreatic cyst was unilocular, filled with runny clear fluid. No solid component or papillary projection was seen within the cyst lining. Microscopically, the cyst was lined by a single layer of cuboidal epithelium, and there was no ovarian-like stroma. The surrounding pancreatic parenchyma was unremarkable. The immunohistochemical stains for the cyst lining were positive for panCK (strong) and EMA (weak). They were negative for calretinin, inhibin, synaptophysin, CD56, chromogranin, CEA and S100. ABPAS and DABPAS staining did not show mucin or glycogen.

\section{References}

1. Bergin D, Ho LM, Jowell PS, et al. Simple pancreatic cysts: CT and endosonographic appearances. AJR Am J Roentgenol 2002; 178: 837-40.

2. Carboni F, Mancini P, Lorusso R, et al. Solitary true cyst of the pancreas in adults. A report of two cases. JOP 2009; 10: 429-31.

\section{DIFFUSE DERMAL ANGIOMATOSIS - A CASE REPORT}

Ashwati Krishnan Varikara, Michael Brown

Department of Anatomical Pathology, ACT Pathology, The Canberra Hospital, Australia

Diffuse dermal angiomatosis (DDA) is usually considered as a variant of reactive angioendotheliomatosis (RAE). Awareness of 